\title{
Long-Term Safety of Rituximab (Risks of Viral and Opportunistic Infections)
}

\author{
Cara D. Varley ${ }^{1,2} \cdot$ Kevin L. Winthrop ${ }^{2,3}$ \\ Accepted: 25 May 2021 / Published online: 16 July 2021 \\ (C) The Author(s), under exclusive licence to Springer Science+Business Media, LLC, part of Springer Nature 2021
}

\begin{abstract}
Purpose of Review We reviewed the current data on infections associated with rituximab use published over the last 5 years. Recent Findings New literature was available on rates of serious infections, Hepatitis B reactivation and screening, and infection with Severe Acute Respiratory Syndrome Coronavirus 2.

Summary Rates of infection varied by study and population, however, higher risk of infection in patients with underlying rheumatologic diseases was seen in those who required a therapy switch, had a smoking history, and those undergoing retreatment who had a serious infection with their first course of therapy. With regards to HBV, the proportion of patients screened continues to be inadequate. Despite the upfront cost, HBV screening and prophylaxis were found to be cost effective. There is still limited data regarding COVID-19 severity in the setting of rituximab, however, rituximab, especially in combination with steroids, may lead to more severe disease and higher mortality.
\end{abstract}

Keywords Rituximab · Hepatitis B · COVID-19 · SARS-CoV-2 · COVID-19 · Infection

\section{Introduction}

Rituximab, a monoclonal antibody targeting CD20, results in B-cell depletion lasting for prolonged periods of time leading to increased risk of infection, especially from infections requiring humoral immunity for control, such as viral infections [1]. Patients receiving rituximab often have immune mediated inflammatory diseases, autoimmune diseases or hematologic malignancies requiring additional chemotherapy or hematopoietic stem cell transplantation. Evaluating risk of infection

This article is part of the Topical Collection on Rheumatoid Arthritis

Cara D. Varley

varleyc@ohsu.edu

Kevin L. Winthrop

winthrop@ohsu.edu

1 Oregon Health \& Science University-Portland State University School of Public Health, Portland, OR, United States

2 Oregon Health \& Science University, School of Medicine, Division of Infectious Diseases, 3181 SW Sam Jackson Park Rd, Mail Code L-457, Portland, OR 97239, United States

3 Oregon Health \& Science University, School of Medicine, Division of Infectious Diseases, Ophthalmology, 181 SW Sam Jackson Park Rd, Mail Code GH104, Portland, OR 97239, United States attributed to rituximab exposure is challenging as these patients often receive other biologic or immunosuppressive agents prior to rituximab, are on concomitant steroids or immunosuppression, may have higher underlying disease activity (in patients with rheumatoid arthritis, for example) or more complications from their underlying condition, all of which can increase the risk of subsequent infections with or without the addition of rituximab. We review the current data on infections associated with rituximab use published over the last 5 years.

\section{General Infection Risk}

Eight studies evaluated the risk of overall infections in those using rituximab. A retrospective, observational study of 70 patients with rheumatologic conditions and greater than 2 years of rituximab use (median 54 months, range 30-138 months) identified infections in $34.3 \%$ resulting in 64 infection-related hospitalizations [2]. Most infections involved the lower respiratory tract and no opportunistic infections were identified [2]. A prospective, observational cohort study of patients on rituximab who had inadequate response to anti-tumor necrosis factor (anti-TNF) agents identified 341 serious infections in 197 patients with an incidence of 8.87/ 100 person-years (95\% confidence interval 7.98-9.86/100 
person-years) [3]. Cumulative rituximab exposure did not increase risk of serious infection with stable infection rates over the 5 years of observation [3]. Observational cohort studies conducted in Sweden and Denmark evaluated incidence of infection at 12 months and 24 months between abatacept, rituximab, tocilizumab, and though rates were highest in the rituximab treated group, no statistically significant difference was found between the three agents $[4,5]$. Patients starting rituximab had more malignancies, longer duration of underlying rheumatologic disease, and were older, which may also explain some of the higher incidence [4]. Of note, current or previous smokers and those who switched between biologic agents as opposed to biologic-naïve patients also had higher incidence of infections [5].

Using the Autoimmunity and Rituximab (AIR) registry, Henry et al. evaluated 1278 patients with rheumatoid arthritis retreated with standard dose rituximab (1000 mg x2) compared to reduced dose $(<2000 \mathrm{mg}$ total $)$ [6]. The reduced dose group had a $39 \%$ reduction in the cumulative rituximab dose per year without a significant difference in European League Against Rheumatism (EULAR) response at year 1 and 2, however, they did have a significant reduction in the rate of serious infections: 2.2/100 person-years in the reduced dose compared to $4.1 / 100$ person-years, $p=0.02$ [6]. In addition, a serious infection during the first course of rituximab was statistically significantly associated with a serious infection during retreatment with a hazard ratio of $3.86,95 \%$ confidence interval 1.82-8.2, $p<0.01$, suggesting closer monitoring of those with infections during their first course of rituximab may be needed [6]. This suggests that reduced doses may provide equal clinical benefit for rheumatoid arthritis with reduced risk of serious infection [6]. Another retrospective cohort study out of Sweden evaluated serious infections and the association with immunoglobulin levels over 5 years in 153 patients with rheumatoid arthritis started on rituximab at either $500 \mathrm{mg}$ or $1000 \mathrm{mg}$ [7]. Infections were seen in $23.1 \%$ at a rate of 7.9/100 person-years and did not correlate with changes in or low immunoglobulin (IgG) levels [7]. In contrast to the study performed by Henry et al., there was no difference in serious infections between different starting doses $[6,7]$. Yusof et al. performed a large retrospective longitudinal study of patients with rheumatic and musculoskeletal diseases with rituximab exposure [8]. They found lower IgG levels ( $<6 \mathrm{gm} /$ liter) at baseline and during therapy to be a consistent predictor of serious infection, suggesting those with decreased immunoglobulin levels at baseline should be monitored closely [8].

The rate of serious infections varied from 2.2 to $9.8 / 100$ person-years in the published studies and did not significantly differ between abatacept, rituximab, tocilizumab [4-7]. Additional risk factors for infection included infection with first treatment of rituximab in those undergoing retreatment, previous or current smoking and switches between biologic therapy, which may indicate treatment failure and increased immunosuppression or side effects that may increase risk of infection $[5,6]$. There is conflicting data on the relationship between rituximab dose on risk of infection, however, dosereduction may be a strategy to consider in a patient with wellcontrolled rheumatoid arthritis and recurrent infections [6, 7].

\section{Hepatitis B (HBV)}

HBV reactivation is a well-known occurrence in patients who are HBV surface antigen $(\mathrm{sAg})$ negative and HBV core antibody positive $(\mathrm{cAb})(8-42 \%)$ or HBVsAg positive (30-60\%) in the setting of rituximab use and can have high morbidity and mortality in this population $[9,10]$. A South Korean study identified HBV reactivation of $7.6 \%$ at 1 year and $10.8 \%$ at 7 years following rituximab initiation in patients with negative HBVsAg and positive HBVcAb [11]. Another study found $10.2 \% \mathrm{HBV}$ reactivation with $40 \%$ mortality in patients with negative HBVsAg and positive $\mathrm{HBVcAb}$, or positive HBVsAg receiving rituximab following renal transplantation [12]. Rituximab-based chemotherapy regimens had a hazard ratio of 11.74 (95\% confidence interval 1.62-84.94, $P=0.02$ ) for HBV reactivation in patients with negative HBVsAg and positive $\mathrm{HBVcAb}$ [13]. Screening for HBV is recommended prior to initiating rituximab $[9,10]$. If $\mathrm{HBV}$ viral load, $\mathrm{HBVsAg}$ or HBVcAb is positive, HBV prophylaxis with entecavir or tenofovir should be initiated and continued for 12 months after discontinuation of rituximab [10]. If patients do not have evidence of prior or active infection with HBV (HBVsAg and HBVcAb negative) and HBV surface antibody $(\mathrm{sAb})$ is negative, vaccination should be considered before initiation of rituximab.

Four studies evaluated routine screening for HBV in the setting of rituximab use. A large retrospective cohort study of the Veterans Health Administration examined HBV testing and treatment in those receiving treatment with an antiCD20 antibody between 2002 and 2014 [14]. They identified 19304 eligible veterans with only $61 \%$ tested for HBVsAg and $73 \%$ tested for $\mathrm{HBVcAb}$ prior to initiating treatment [14]. Of those who were positive and at high risk for infection, less than $37 \%$ received HBV antiviral agents during antiCD20 antibody administration and $17 \%$ or less received HBV vaccination before anti-CD20 antibody administration [14]. Less than $2 \%$ had HBV viral loads completed while on anti-CD20 antibody therapy, limiting detection of HBV reactivation in this population [14]. Paul et al evaluated HBV screening rates by specialty, identifying only $48 \%$ of patients on rituximab with $\mathrm{HBV}$ sAg or $\mathrm{HBV} \mathrm{cAb}$ completed 2 months before or 1 month after immunosuppressive therapy initiation [15]. Screening rates were lowest among gastroenterologists (33\%), followed by oncologists $(37 \%)$, rheumatologists (54\%), and transplant specialists (85\%) [15]. Having a 
transplant specialty provider, Asian race, male gender, younger age, and treatment after 2008 were significantly associated with being screened for HBV [15]. A more recent study of HBV screening at two health systems in San Francisco performed between 2012 and 2016 identified $61 \%$ at the university system and $90 \%$ at the safety net system receiving adequate HBV screening prior to rituximab initiation [16]. Trends were similar in antiviral prophylaxis prescribing for patients at risk for HBV reactivation, with only $66 \%$ and $92 \%$ of patients at the university and safety net systems, respectively [16].

Another study out of the United Kingdom evaluated educational strategies to increase HBV screening prior to rituximab use and identified only $23 \%$ and $19 \%$ of patients had testing for HBVsAg and HBVcAb, respectively [17]. Following this evaluation, new hospital HBV screening guidelines were emailed to all providers, along with 30-min education sessions targeting clinicians prescribing rituximab, and the laboratory reporting algorithm was revised for this high-risk population [17]. Following these interventions, the proportion of patients screened increased to $79 \%$ for $\mathrm{HBVsAg}$ and $78 \%$ for $\mathrm{HBVcAb}$, indicating simple interventions can improve screening in this population [17].

Crespo et al. evaluated the cost effectiveness of screening for HBV reactivation and administering prophylaxis with tenofovir disoproxil fumarate prior to R-CHOP (rituximab, cyclophosphamide, doxorubicin, vincristine, and prednisone) and identified they would prevent $7.36 \mathrm{HBV}$ reactivations in a cohort of 1000 people [18]. The cost of screening and prophylaxis was an additional $€ 135$ per patient with a savings of $€ 18,376$ per prevented reactivation, suggesting this is a costeffective approach in this population [18].

A study performed in Hong Kong evaluating a novel biomarker, Hepatitis B core-related antigen (HBcrAg), in negative $\mathrm{HBVsAg}$ and positive $\mathrm{HBVcAb}$ patients undergoing rituximab or stem cell transplantation identified baseline HBcrAg positivity as a risk factor for two-year HBV reactivation with a hazard ration of 2.94 (95\% confidence interval 1.43-6.07) [19]. A pilot study examining cytokine single nucleotide polymorphisms (SNPs) associated with HBV reactivation (HBsAg in the serum and an HBV viral load $>2000$ $\mathrm{IU} / \mathrm{mL}$ ) in a group of 104 patients with B-cell non-Hodgkin lymphoma who were HBVsAg negative and unvaccinated for HBV [20]. SNPs in IL18 (rs243908) and an IL4 haplotype (rs2243248 rs2243263), along with greater than 6 cycles of rituximab were statistically significantly associated with HBV reactivation [20]. Both hold promise for improved identification of patients at risk for HBV reactivation along with more targeted methods of antiviral prophylaxis.

Overall, screening for HBV in patients prior to receiving rituximab continues to be inadequate, however, targeted education, hospital-specific guidelines and revised laboratory reporting for patients initiating rituximab are strategies that can improve screen rates. Despite the upfront cost, HBV screening and prophylaxis were found to be cost effective [18]. In addition, novel biomarkers and genetic testing may provide better identification and ability to provide more targeted antiviral prophylaxis for those at highest risk of HBV reactivation.

\section{Severe Acute Respiratory Syndrome Coronavirus 2 (SARS-CoV-2)}

The effect of rituximab on COVID-19 remains uncertain, however, concerns are raised regarding both viral clearance, increased disease severity and inability to form antibodies protective from future infection $[1,21]$. Limited data are available on the on the severity of COVID-19 in those with rheumatologic diseases and fewer address the effects of rituximab exposure. Early studies demonstrated no increase in hospitalization rates or severe disease between those with rheumatologic diseases compared to the general population [22, 23]. In one of the largest studies performed by the COVID-19 Global Rheumatology Alliance physician-reported registry, 231 out of 600 patients with rheumatologic diseases and COVID-19 were taking biologic therapy [24]. Half the patients required inpatient admission and 9\% died [24]. Biologic therapy was not associated with an increased risk of hospitalization, however, only 27 (12\%) were taking an anti-CD-20 agent, limiting evaluation of severe disease risk in this group of patients [24]. Nuno et al. evaluated a cohort of patients with COVID-19 in addition to underlying rheumatic disease and found $56.6 \%$ required hospital admission and $11.5 \%$ died [25]. Biologic therapy was not associated with hospitalization or death, however, all patients $(n=7)$ on rituximab required inpatient admission and one died [25]. Similarly, a small observation study of 41 patients with rheumatologic disease and COVID-19 in Spain found $68.3 \%$ required hospital admission, $14.6 \%$ intensive care unit (ICU) admission, and 7.3\% died [26]. Only three patients were taking anti-CD20 monoclonal antibodies, including one of the fatalities, a 91-year-old woman with hypertension and vasculitis [26].

A large registry of 3729 patients with rheumatic disease and presumed or confirmed COVID-19 infection looked at risk for COVID-19 related deaths [27]. They identified age, male sex, hypertension, cardiovascular disease, chronic lung disease, higher disease activity, immunosuppressants, absence of disease-modifying antirheumatic drugs, prednisone $\geq 10 \mathrm{mg} /$ day, and rituximab as independent risk factors for death with the highest odds ratio (OR) associated with rituximab exposure (OR 4.04, 95\% confidence interval 2.32-7.03) [27]. A retrospective cohort study performed in the Kaiser Permanente health system in California assessed patients with multiple sclerosis taking rituximab and identified a higher risk of COVID-19 hospitalization, but not death or infection, compared to the general population without multiple sclerosis 
[28]. Of note, COVID-19 hospitalizations decreased as the time from last rituximab infusion increased, with no hospitalizations when COVID-19 occurred $>6$ months after the last rituximab infusion [28]. A rituximab dose of $>1000 \mathrm{mg}$ was also associated with hospitalization $(\mathrm{OR}=6.24,95 \%$ confidence interval 1.38-28.31) [28]. Prednisone use of $\geq 5 \mathrm{mg} /$ day or $\geq 10 \mathrm{mg}$ /day prior to COVID-19 diagnosis was identified as a risk factor for hospital admission and death in multiple studies, however, the number of comorbidities and concomitant immunosuppressive therapies were also high in these populations $[24,25,27]$. The reliability of using hospitalization as an endpoint for severity is questionable, as many of these patients may be more likely to be admitted due to their immunosuppression and concern for higher morbidity and mortality with COVID-19, especially early in the pandemic.

A case series from Spain reported mild COVID-19 infections with only one hospitalization in patients taking antiCD20 with multiple sclerosis, however, SARS-CoV2 was diagnosed only by symptoms [29]. In contrast, a case series in Tehran evaluated COVID-19 in nine patients with multiple sclerosis taking rituximab with five requiring hospitalization, two of which required ICU admission and two died, giving a mortality of $40 \%$ for those admitted [30]. None of the patients reported steroid use prior to their COVID-19 diagnosis [30]. A case series in Spain evaluated 13 patients with probable or confirmed COVID-19 treated with rituximab in the 12 months before the study [31]. Patients had rheumatoid arthritis, systemic vasculitis, Sjogren syndrome or systemic lupus erythematosus as their underlying rheumatologic condition and many were on additional immunosuppressive agents including prednisone $(53.8 \%)$, methotrexate $(46.2 \%)$, and hydroxychloroquine $(7.7 \%)$ [31]. Eight $(61.5 \%)$ required hospitalization, five met criteria for acute respiratory distress syndrome (ARDS), and three died (23.1\%) [31].

A case report by Guilpan et al. describes severe COVID-19 pneumonia with recovery at day 25 of infection in a patient with granulomatosis with polyangiitis treated with rituximab and prednisone [1]. Another case reviews a gentleman with granulomatosis with polyangiitis, also on rituximab and prednisone complicated by hypogammaglobulinemia, with initially mild symptoms who required readmission on day 14 of illness with increasing oxygen requirements [21]. SARS$\mathrm{CoV} 2$ remained detectable in the nasopharyngeal swab for almost 30 days, with his first negative test at day 32 following the onset of symptoms [21]. Two case reports were presented from Germany, however, prednisone use was not discussed in either report [32]. One patient with relapsing-remitting multiple sclerosis had a mild disease course with no detectable B cells on hospital admission and negative SARS-CoV-2 antibodies 8 weeks after she tested positive by PCR [32]. The second patient had multiple underlying comorbidities with a more severe illness and prolonged ICU course complicated by
ARDS, renal failure, cardia arrhythmia, and bacterial infection, with eventual recovery after 6 weeks of hospitalization. The second patient had detectable but suppressed B cells but did develop SARS-CoV-2 antibodies [32]. An additional two fatal COVID-19 cases from Germany were described with prolonged SARS-CoV-2 viremia, with the viral load peaking just prior to death, in patients receiving rituximab for diffuse large B-Cell lymphoma and mantle cell lymphoma [33]. One of these patients was taking steroids [33]. Two more patients taking rituximab for rheumatoid arthritis were reported from Germany with fatal outcomes [34]. One was taking $5 \mathrm{mg} /$ day of prednisone as well as methotrexate with rituximab infusion 6 months prior to her COVID-19 diagnosis. The other patient, also on methotrexate, had his last rituximab infusion, including $50 \mathrm{mg}$ prednisolone premedication, 2 weeks before his COVID-19 diagnosis [34]. Another case report from Oregon reviews a patient with granulomatosis with polyangiitis on rituximab with multiple ongoing COVID-19 exposures who acquired SARS-CoV-2 twice [35]. One month after her second infection, COVID-19 IgGfig levels were undetectable [35].

Based on the limited available data, there is concern that rituximab, especially in combination with high-dose steroids, may lead to more severe disease and increased risk of death with COVID-19. The numbers are small, but over half of those with COVID-19 and rheumatologic conditions are admitted, with two case series specifically evaluating rituximab use identifying over half of those admitted meeting criteria for ARDS and 37-40\% mortality [24-28, 31]. We currently have limited data on vaccine response in this population with one study showing decreased antibody response after a single dose of SARS-CoV-2 mRNA vaccine in those taking methotrexate or rituximab [36]. We do have data with other vaccines demonstrating reduced humoral response in the setting for rituximab [37]. As with other vaccines, one should administer SARS-CoV-2 vaccine as far as possible from their last rituximab dose and would avoid rituximab administration for 2-4 weeks after vaccination until more clinical data are available $[37,38]$. Given this, we would continue to encourage ongoing public health measures such as mask wearing, social distancing, hand hygiene, and potential vaccination of close contacts given early data showing decreased transmission and viral load following vaccination [39-42].

\section{Conclusions}

The rate of serious infections in patients receiving rituximab varied from 2.2 to $9.8 / 100$ person-years in the studies published over the last 5 years and did not significantly differ between abatacept, rituximab, tocilizumab in those with underlying rheumatic diseases [2-8]. Additional risk factors for infection included infection with first treatment of rituximab 
in those undergoing retreatment, previous or current smoking, switches between biologic therapy, which may help identify patients who require closer monitoring $[4,5]$. With regards to HBV, screening continues to be inadequate, however, targeted education, hospital-specific guidelines, and revised laboratory reporting for patients initiating rituximab are strategies that can improve screen rates. Despite the upfront cost, HBV screening and prophylaxis were found to be cost effective [18]. There is still limited data regarding COVID-19 severity in the setting of rituximab, however, case reports, case-series, and a larger observational study suggest rituximab use is associated with more severe disease, higher mortality with COVID-19, and hospitalizations appear to be higher within the first 6 months following rituximab dose [24-28, 31]. We currently have limited data on vaccine response in this population with one study showing decreased antibody response after a single dose of SARS-CoV-2 mRNA vaccine in those taking methotrexate or rituximab [36]. Given this, we would continue to encourage ongoing public health measures such as mask wearing, social distancing, hand hygiene, and potential vaccination of close contacts given early data showing decreased transmission and viral load following vaccination [39-42].

\section{Declarations}

Conflict of Interest Cara Varley declares that she has no conflict of interest.

Kevin Winthrop has received research support from BMS, Pfizer.

Kevin Winthrop serves as a consultant for Pfizer, AbbVie, Union Chimique Belge (UCB), Eli Lilly \& Company, Galapagos, GlaxoSmithKline (GSK), Roche, Gilead, BMS, Regeneron, Sanofi, AstraZeneca, Novartis.

\section{References}

1. Guilpain P, Le Bihan C, Foulongne V, Taourel P, Pansu N, Maria ATJ, et al. Rituximab for granulomatosis with polyangiitis in the pandemic of covid-19: lessons from a case with severe pneumonia. Ann Rheum Dis. 2021;80(1):e10.

2. Vikse J, Jonsdottir K, Kvaloy JT, Wildhagen K, Omdal R. Tolerability and safety of long-term rituximab treatment in systemic inflammatory and autoimmune diseases. Rheumatol Int. 2019;39(6):1083-90.

3. Winthrop KL, Saag K, Cascino MD, Pei J, John A, Jahreis A, et al. Long-Term Safety of Rituximab in Rheumatoid Arthritis: Analysis From the SUNSTONE Registry. Arthritis care \& research. 2018.

4. Gron KL, Arkema EV, Glintborg B, Mehnert F, Ostergaard M, Dreyer L, et al. Risk of serious infections in patients with rheumatoid arthritis treated in routine care with abatacept, rituximab and tocilizumab in Denmark and Sweden. Ann Rheum Dis. 2019;78(3): 320-7.

5. Gron KL, Glintborg B, Norgaard M, Mehnert F, Ostergaard M, Dreyer L, et al. Overall infection risk in rheumatoid arthritis during treatment with abatacept, rituximab and tocilizumab; an observational cohort study. Rheumatology (Oxford, England). 2020;59(8):1949-56.

6. Henry J, Gottenberg J-E, Rouanet S, Pavy S, Sellam J, Tubach F, et al. Doses of rituximab for retreatment in rheumatoid arthritis: influence on maintenance and risk of serious infection. Rheumatology (Oxford, England). 2018;57(3):538-47.

7. Einarsson JT, Evert M, Geborek P, Saxne T, Lundgren M, Kapetanovic MC. Rituximab in clinical practice: dosage, drug adherence, Ig levels, infections, and drug antibodies. Clin Rheumatol. 2017;36(12):2743-50.

8. Md Yusof MY, Vital EM, McElvenny DM, Hensor EMA, Das S, Dass S, et al. Predicting severe infection and effects of hypogammaglobulinemia during therapy with rituximab in rheumatic and musculoskeletal diseases. Arthritis Rheumatol. 2019;71(11):1812-23.

9. Law MF, Ho R, Cheung CKM, Tam LHP, Ma K, So KCY, et al. Prevention and management of hepatitis $\mathrm{B}$ virus reactivation in patients with hematological malignancies treated with anticancer therapy. World J Gastroenterol. 2016;22(28):6484-500.

10. Terrault NA, Lok ASF, McMahon BJ, Chang K-M, Hwang JP, Jonas $\mathrm{MM}$, et al. Update on prevention, diagnosis, and treatment of chronic hepatitis B: AASLD 2018 hepatitis B guidance. Hepatology. 2018;67(4):1560-99.

11. Lee HL, Jang JW, Han JW, Lee SW, Bae SH, Choi JY, et al. Early hepatitis B surface antigen seroclearance following antiviral treatment in patients with reactivation of resolved hepatitis B. Dig Dis Sci. 2019;64(10):2992-3000.

12. Lee J, Park JY, Huh KH, Kim BS, Kim MS, Kim SI, et al. Rituximab and hepatitis B reactivation in HBsAg-negative/ antiHBc-positive kidney transplant recipients. Nephrol Dial transplant: official publication of the European Dialysis and Transplant Association - European Renal Association. 2017;32(4):722-9.

13. Su Y-C, Lin P-C, Yu H-C, Wu C-C. Hepatitis B virus reactivation in patients with resolved hepatitis $\mathrm{B}$ virus infection receiving chemotherapy or immunosuppressive therapy. Eur J Gastroenterol Hepatol. 2018;30(8):925-9.

14. Hunt CM, Beste LA, Lowy E, Suzuki A, Moylan CA, Tillmann HL, et al. Veterans health administration hepatitis B testing and treatment with anti-CD20 antibody administration. World J Gastroenterol. 2016;22(19):4732-40.

15. Paul S, Shuja A, Tam I, Kim EM, Kang S, Kapulsky L, et al. Gastroenterologists have suboptimal hepatitis B virus screening rates in patients receiving immunosuppressive therapy. Dig Dis Sci. 2016;61(8):2236-41.

16. Schmajuk G, Tonner C, Trupin L, Li J, Sarkar U, Ludwig D, et al. Using health-system-wide data to understand hepatitis B virus prophylaxis and reactivation outcomes in patients receiving rituximab. Medicine. 2017;96(13):e6528.

17. Dyson JK, Jopson L, Ng S, Lowery M, Harwood J, Waugh S, et al. Improving testing for hepatitis B before treatment with rituximab. Eur J Gastroenterol Hepatol. 2016;28(10):1172-8.

18. Crespo J, Esteban R, Torres C, Oyaguez I, Casado MA, Buti M. Cost-effectiveness of a hepatitis B virus screening strategy to prevent reactivation in patients with hematologic neoplasms. Rev Esp Enferm Dig: organo oficial de la Sociedad Espanola de Patologia Digestiva. 2017;109(9):619-26.

19. Seto W-K, Wong DK-H, Chan TS-Y, Hwang Y-Y, Fung J, Liu KS$\mathrm{H}$, et al. Association of Hepatitis B Core-Related Antigen With Hepatitis B Virus Reactivation in Occult Viral Carriers Undergoing High-Risk Immunosuppressive Therapy. Am J Gastroenterol. 2016;111(12):1788-95.

20. Hsiao L-T, Wang H-Y, Yang C-F, Chiou T-J, Gau J-P, Yu Y-B, et al. Human Cytokine Genetic Variants Associated With HBsAg Reverse Seroconversion in Rituximab-Treated Non-Hodgkin Lymphoma Patients. Medicine. 2016;95(11):e3064. 
21. Leipe J, Wilke EL, Ebert MP, Teufel A, Reindl W. Long, relapsing, and atypical symptomatic course of COVID-19 in a B-cell-depleted patient after rituximab. Semin Arthritis Rheum. 2020;50(5):10878.

22. Haberman R, Axelrad J, Chen A, Castillo R, Yan D, Izmirly P, et al. Covid-19 in Immune-Mediated Inflammatory Diseases - Case Series from New York. N Engl J Med. 2020;383(1):85-8.

23. Pablos JL, Galindo M, Carmona L, Lledó A, Retuerto M, Blanco R, et al. Clinical outcomes of hospitalised patients with COVID-19 and chronic inflammatory and autoimmune rheumatic diseases: a multicentric matched cohort study. Ann Rheum Dis. 2020;79(12): 1544-9.

24. Gianfrancesco M, Hyrich KL, Al-Adely S, Carmona L, Danila MI, Gossec L, et al. Characteristics associated with hospitalisation for COVID-19 in people with rheumatic disease: data from the COVID-19 Global Rheumatology Alliance physician-reported registry. Ann Rheum Dis. 2020;79(7):859-66.

25. Nuño L, Novella Navarro M, Bonilla G, Franco-Gómez K, Aguado $\mathrm{P}$, Peiteado D, et al. Clinical course, severity and mortality in a cohort of patients with COVID-19 with rheumatic diseases. Ann Rheum Dis. 2020;79(12):1659-61.

26. Sanchez-Piedra C, Diaz-Torne C, Manero J, Pego-Reigosa JM, Rua-Figueroa I, Gonzalez-Gay MA, et al. Clinical features and outcomes of COVID-19 in patients with rheumatic diseases treated with biological and synthetic targeted therapies. Ann Rheum Dis. 2020;79(7):988-90.

27. Strangfeld A, Schäfer M, Gianfrancesco MA, Lawson-Tovey S, Liew JW, Ljung L, et al. Factors associated with COVID-19related death in people with rheumatic diseases: results from the COVID-19 Global Rheumatology Alliance physician-reported registry. Annals of the rheumatic diseases. 2021:annrheumdis-2020219498.

28. Langer-Gould A, Smith JB, Li BH, Group KMS. Multiple sclerosis, rituximab, and COVID-19. Ann clin and transl neurol. 2021;8(4): 938-43.

29. Montero-Escribano P, Matías-Guiu J, Gómez-Iglesias P, PortaEtessam J, Pytel V, Matias-Guiu JA. Anti-CD20 and COVID-19 in multiple sclerosis and related disorders: A case series of $60 \mathrm{pa}-$ tients from Madrid, Spain. Mult Scler Relat Disord. 2020;42: 102185.

30. Esmaeili S, Abbasi MH, Abolmaali M, Mojtahed M, Alavi SNR, Soleimani S, et al. Rituximab and risk of COVID-19 infection and its severity in patients with MS and NMOSD. BMC Neurol. 2021;21(1): 183 .

31. Loarce-Martos J, Garcia-Fernandez A, Lopez-Gutierrez F, GarciaGarcia V, Calvo-Sanz L, Del Bosque-Granero I, et al. High rates of severe disease and death due to SARS-CoV-2 infection in rheumatic disease patients treated with rituximab: a descriptive study. Rheumatol Int. 2020;40(12):2015-21.

32. Woo MS, Steins D, Hausler V, Kohsar M, Haag F, Elias-Hamp B, et al. Control of SARS-CoV-2 infection in rituximab-treated neuroimmunological patients. J Neurol. 2021;268(1):5-7.

33. Tepasse P-R, Hafezi W, Lutz M, Kuhn J, Wilms C, Wiewrodt R, et al. Persisting SARS-CoV-2 viraemia after rituximab therapy: two cases with fatal outcome and a review of the literature. $\mathrm{Br} \mathrm{J}$ Haematol. 2020;190(2):185-8.

34. Schulze-Koops H, Krueger K, Vallbracht I, Hasseli R, Skapenko A. Increased risk for severe COVID-19 in patients with inflammatory rheumatic diseases treated with rituximab. Ann Rheum Dis. 2021;80(5):e67.

35. Friedman MA, Winthrop KL. Second COVID-19 infection in a patient with granulomatosis with polyangiitis on rituximab. Ann Rheum Dis. 2021

36. Boyarsky BJ, Ruddy JA, Connolly CM, Ou MT, Werbel WA, Garonzik-Wang JM, et al. Antibody response to a single dose of SARS-CoV-2 mRNA vaccine in patients with rheumatic and musculoskeletal diseases. Annals of the rheumatic diseases. 2021: annrheumdis-2021-220289.

37. Friedman MA, Winthrop KL. Vaccines and Disease-Modifying Antirheumatic Drugs: Practical Implications for the Rheumatologist. Rheum Dis Clin N Am. 43(1):1-13.

38. Waldman RA, Creed M, Sharp K, Adalsteinsson J, Imitola J, Durso $\mathrm{T}$, et al. Toward a COVID-19 vaccine strategy for patients with pemphigus on rituximab. J Am Acad Dermatol. 2021;84(4):e197e8.

39. Levine-Tiefenbrun M, Yelin I, Katz R, Herzel E, Golan Z, Schreiber L, et al. Decreased SARS-CoV-2 viral load following vaccination. medRxiv. 2021:2021.02.06.21251283.

40. Tande AJ, Pollock BD, Shah ND, Farrugia G, Virk A, Swift M, et al. Impact of the COVID-19 vaccine on asymptomatic infection among patients undergoing pre-procedural COVID-19 molecular screening. Clin Infect Dis. 2021.

41. V Shah AS, Gribben C, Bishop J, Hanlon P, Caldwell D, Wood R, et al. Effect of vaccination on transmission of COVID-19: an observational study in healthcare workers and their households. medRxiv. 2021:2021.03.11.21253275.

42. Varley CD, Ku JH, Winthrop KL. COVID-19 pandemic management and the rheumatology patient. Best Pract Res Clin Rheumatol. 2021;35(1):101663.

Publisher's Note Springer Nature remains neutral with regard to jurisdictional claims in published maps and institutional affiliations. 